\title{
WHAT CAN WE LEARN FROM CHARMLESS RARE B DECAYS - THE PAST/NEXT 3 YEARS
}

\author{
GEORGE W.S. HOU \\ Department of Physics, National Taiwan University, Taipei, Taiwan, R.O.C.
}

A personal perspective is given on physics of charmless rare B decays: $1997-2003$.

\section{Prelude: Berkeley to Osaka}

My first ICHEP was Berkeley 1986, where I heard about CLEO limits on rare B decays. A host of strong (P, e.g. $B \rightarrow \phi K^{+}$), radiative or electromagnetic (EMP, e.g. $B \rightarrow K^{*} \gamma$ ) and electroweak (EWP, e.g. $B \rightarrow K \ell^{+} \ell^{-}$) modes were given, with limits typically a few $\times 10^{-4}$, but EMP limits were only $\sim 10^{-3}$, reflecting the time before the advent of CsI-based EM calorimetry. 1

Though still contending with limits, I embarked on a study of these modes, encountering (and missing) a few surprises. I found that the $Z$ penguin dominated the EWP's. 1 While obvious for $b \rightarrow s \nu \bar{\nu}$, for $b \rightarrow s \ell^{+} \ell^{-}$the EMP had naively been assumed to be dominant. Although I missed the "large QCD corrections" to EMP that enhanced $b \rightarrow s \gamma$ to a few $\times 10^{-4}$, I did uncover the sensitivityl to $H^{+}$effect, which later lead to the stringent bound on $m_{H^{+}}$in SUSY type of two Higgs models. I encountered the surprise of "higher order dominance" that $b \rightarrow s g^{*} \rightarrow s \bar{q} q>b \rightarrow s g$. Much of the above has to do with GIM cancellation subtleties or the non-decoupling of $m_{t}$ in SM.

Why did I bother only with inclusive processes at that time? Well, these can be, by argument of duality, viewed as quark level processes hence dominated by S.D. physics ( virtual collider). But for exclusive processes, hadronization brings in L.D. physics that is likely to blur S.D. information. Unless, ... ugh, ... one has factorization!? Factorization means separating the product of quark currents and forming hadronic matrix elements of bilinears. Naively, for $b \rightarrow d \bar{u} u$ to mediate $\bar{B} \rightarrow \pi^{-} \pi$, the $\bar{d} u$ current created by virtual $W$ projects directly into a pion (decay constant), while the $b \rightarrow u$ current mediates $B \rightarrow \pi$ transition (form factor). That is, one assumes (wishes)

$$
\langle\pi \pi|(\bar{d} u)(\bar{u} b)| B\rangle \stackrel{\text { fac. }}{\sim}\langle\pi|\bar{d} u| 0\rangle\langle\pi|\bar{u} b| B\rangle .
$$

But this was too big an "if" for me. Despite the ease for detection, I could not bring myself to believe in factorization, and hence never worked on exclusive

$\bar{a}$ The first penguin, $B \rightarrow K^{*} \gamma$, emerged $\sim 5 \times 10^{-5}$ soon after CLEO-II (w/ CsI) started. 
modes ... until I had a data-driven conversion experience at end of 1998 !

Strong penguins have emerged en masse since 1997 at CLEO, where $10^{7}$ $B \bar{B}$ 's have been collected, and at Osaka 2000, Belle and BaBar both reported first results with comparable amounts of data, collected in first year of running! The four $K \pi$ modes and relatively small $\pi^{+} \pi^{-}$mode observed by CLEO are confirmed by Belle, but BaBar reports a higher (lower) $\pi^{+} \pi^{-}\left(K^{+} \pi^{-}\right)$. More data is needed, and certainly expected, but Golutvin 4 already states: "Present data favour large $\arg V_{u b}$ and FSI." We shall address why this is so, but let me step a few years back to the time of the emerging strong penguins.

\section{Experimental Surprise of $\eta^{\prime} X_{s}$ and $\eta^{\prime} K$}

With a few $\times 10^{6} B \bar{B}$ 's, CLEO announced in 1997 that $B \rightarrow \eta^{\prime} X_{s} \sim 6 \times 10^{-4}$ for $2<p_{\eta^{\prime}}<2.7 \mathrm{GeV}$, and $\eta^{\prime} K$ a tenth less. This was not predicted by any theorist. Many models and speculations ensued. It is puzzling that, with $\eta^{\prime}$ the heaviest and stickiest (gluev) member of the $0^{-}$nonet, how can it come so "fast"? One interesting idea, 5 the only one so far that can explain the $m_{X_{s}}$ spectrum, links $\eta^{\prime}$ production to the gluon anomaly: The derivative coupling nature of the $g^{*}-g-\eta^{\prime}$ anomaly vertex spits out $\eta^{\prime}$ with high momentum in $b \rightarrow s g^{*} \rightarrow s g \eta^{\prime}$. A criticism is that such coupling must be cut off by some form factor. However, the $g^{*} g$ channel has $0^{-+}$quantum numbers, and the high glueball mass scale $(\sim 2.5 \mathrm{GeV}$ from lattice) may well delay the form factor suppression. One way 6 to check this is to study $Z \rightarrow q \bar{q} g+\eta^{\prime}$.

The $\eta^{\prime} K$ analysis of CLEO is now very robust, and is starting to be checked by Belle/BaBar, but inclusive study has not yet been updated by any group (even CLEO). This is certainly a volatile area where more insight, if not surprises, can be attained. We still lack a clear theory.

\section{The Path to $\gamma>90^{\circ}$ and Factorization (and FSI?)}

\section{Factorization and $\gamma \gtrsim \mathbf{9 0 ^ { \circ }}$}

CLEO data has driven phenomenology in a fine way in the last 3 years 1

$1997: \bar{K}^{0} \pi^{-}>K^{-} \pi^{+}$— This lead to the Fleischer-Mannel bound (though $\bar{K}^{0} \pi^{-}$was just above $3 \sigma$ ), and a boom in theory work, eventually leading to model-independent methods for extracting $\gamma$.

$1998: K^{-} \pi^{0} \gtrsim \bar{K}_{0}^{0} \pi^{-} \simeq K^{-} \pi^{+} \simeq 1.4 \times 10^{-5}-K^{0} \pi \simeq K \pi$ prompted the first suggestionl for large $\gamma$; surprising strength of $K \pi^{0}$ indicated EWP. 
$1999: \rho^{0} \pi^{-}, \rho^{ \pm} \pi^{\mp}, \omega \pi(\omega K$ disappear $) —$ Evidence for $b \rightarrow u$ tree $(\mathrm{T})$. $K \pi^{0} \simeq \frac{2}{3}\left(\bar{K}^{0} \pi \simeq K \pi\right)$ Fine, EWP at work

$\pi \pi \sim \frac{1}{4} K \pi-$ Further indication for large $\gamma$.

$K^{0} \pi^{0} \sim K \pi, K^{0} \pi \Longrightarrow$ Problem 10

The host of emerging modes lead to the observation that,

Factorization works in two body charmless rare $B$ decays, if $\cos \gamma \lesssim 0$

It even lead to11 a "global (rare B) fit" of more than 10 modes that gave $\gamma \simeq 105^{\circ}$, seemingly in some conflict with the well-known "CKM Fit" value of $\gamma \simeq 60^{\circ}$. Sufficie it to say that, by end of 1999 , all B practioners had switched to $\gamma \gtrsim 80^{\circ}-90^{\circ}$, as reflected in the 5 rare $\mathrm{B}$ theory talks at Osaka. This could have harbingered the lower central value of $\sin 2 \beta$ seen by BaBar/Belle this summer, although dust is far from settled.
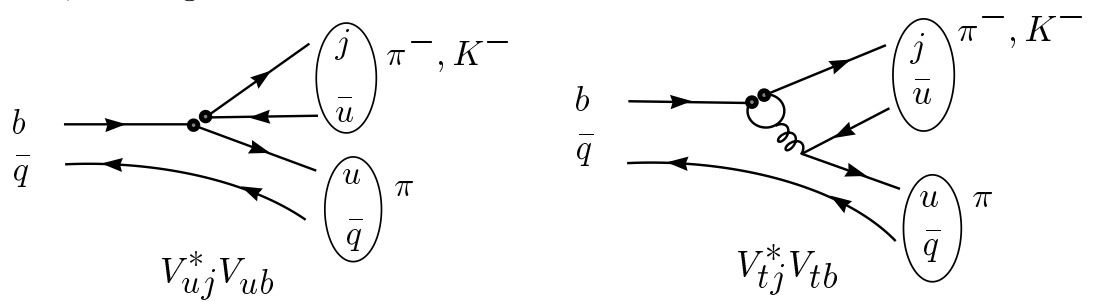

What is the physics effect? Let us illustrate with $K \pi$ vs. $\pi \pi$. With only $\mathrm{T}$ contributions, $\left|V_{u s}\right|^{2} \ll\left|V_{u d}\right|^{2}$ implies $K \pi \ll \pi \pi$. Thus, the observed $K \pi \gtrsim \pi \pi$ implies $\mathrm{P}$ dominance in $K \pi_{\mathrm{m}}$ and substantial "penguin pollution" to $\pi \pi$. As data refined, it was realized 1 that T-P interference contains more information. As $V_{u s}^{*} V_{u b}=\lambda\left|V_{u b}\right| e^{-i \gamma}=\lambda V_{u d}^{*} V_{u b}$ has the same phase, while the real part of $V_{t s}^{*} V_{t b} \cong-\left|V_{c b}\right|$ and $V_{t d}^{*} V_{t b} \cong \lambda\left|V_{c b}\right|\left[1-\sqrt{\rho^{2}+\eta^{2}} e^{-i \gamma}\right]$ have opposite sign since $\sqrt{\rho^{2}+\eta^{2}} \equiv\left|V_{u b}\right| / \lambda\left|V_{c b}\right| \simeq 0.4$, hence T-P interference is anticorrelated in $K \pi$ vs. $\pi \pi$. Thus, $K \pi \Uparrow$ implies $\pi \pi \Downarrow$, and vice versa. 9

\section{FSI?}

A problem was already apparent by summer 1999: $K^{0} \pi^{0}$ seems too large 10 (Again a chorus line of theorists at Osaka)! As mentioned, $K \pi^{0} / K \pi \simeq 0.65$ confirms constructive EWP-P interference for $K \pi^{0}$ in SM. From the operators and the $\pi^{0}$ w.f. (change from $u \bar{u}$ to $d \bar{d}$ ) one expects destructive EWP-P interference in $K^{0} \pi^{0}$, hence $K^{0} \pi^{0}>K \pi^{0}$ is very hard to reconcile.

We proposed a half-way solution, resorting to large final state interaction (FSI) phases. 10 If we start with e.g. $\gamma=110^{\circ}$, then $K \pi: K^{0} \pi: K \pi^{0}$ :

\footnotetext{
${ }^{b}$ Only the sign change in $\cos \gamma$ was conservatively advocated in original paper.
} 
$K^{0} \pi^{0}=1: 0.94: 0.65: 0.35$, as compared to the experimental (CLEO only) $1: 1.06: 0.67: \mathbf{0 . 8 5}$. Allowing $\delta_{K \pi}$ (strong phase difference between $I=\frac{3}{2}$ and $\frac{1}{2}$ amplitudes) to be $\sim 90^{\circ}$, we find the ratio becomes $1: 1.12: 0.61: 0.47$. This is far from resolving the problem, but it is in the right direction. What's more, we find that $\pi \pi<\pi \pi^{0}$ can be achieved (taking $\delta_{\pi \pi} \sim \delta_{K \pi}$ ) and central values for $a_{\mathrm{CP}}$ in $K \pi, K^{0} \pi, K \pi^{0}$ modes become "just right", and there are further dramatic consequences:

- $\pi^{0} \pi^{0} \sim \pi \pi \sim 3-5 \times 10^{-6} \lesssim \pi \pi^{0}$ (still satisfy CLEO bound).

- $a_{\mathrm{CP}}^{\bar{K}^{0} \pi^{0}} \sim-a_{\mathrm{CP}}^{K^{-} \pi^{0}}$ large; $a_{\mathrm{CP}}^{\pi \pi}, a_{\mathrm{CP}}^{\pi^{0} \pi^{0}}$ as large as $\sim-60 \%,-30 \%$ possible. These would be measurable in a couple of years.

So now we have an oxymoron: Factorization works, but FSI is large. Our view is, however, phenomenological: Data indicates that factorization works for the first 10-20 or so two body rare B modes. The $\delta_{K \pi}$ and $\delta_{\pi \pi}$ phases are the minimal extension of parameters allowed in the factorization framework. We do not pretend to know their origin. They could be effective parameters arising from e.g. annihilation diagrams. But if they genuinely arise from L.D. physics, they would then pose a real problem for PQCD.

\section{New Physics: Probing Flavor and/or CP Violation}

As a "virtual collider", B decays and mixings provide a natural hunting ground for New Physics, esp. flavor and CP violation. Let us illustrate with SUSY.

It is known that $\tilde{g}-\tilde{q}$ loops could easily generate $F_{2}^{\mathrm{NP}} \bar{s} i \sigma_{\mu \nu} m_{b} R b G^{\mu \nu}$ type couplings, and also $b_{L} \rightarrow s_{R}$ chirality flips that are absent in SM. For example, $a_{\mathrm{CP}}$ in inclusive $B \rightarrow \eta^{\prime}+X_{s}$ at $10 \%$ level 8 A late 1997 rumor that CLEO had $a_{\mathrm{CP}}(K \pi) \sim 100 \%$ led us to put in a sizable $F_{2}^{\mathrm{NP}}$ and managed 12 to yank $a_{\mathrm{CP}}$ 's up to $50 \%$. Unfortunately, the rumor ended with CLEO 1999 direct $a_{\mathrm{CP}}$ results in 5 modes, all consistent with zero with errors $\sim 20 \%$.

Along a different line, we compiled 3 measurables which could test $b_{L} s_{R} \gamma$ couplings that would definitely indicate New Physics: mixing dependent and direct CP violation in $B \rightarrow K^{*} \gamma$, and $\Lambda$ polarization in $\Lambda_{b} \rightarrow \Lambda \gamma$ decay.

It turns out that $b \rightarrow d$ penguins may be more promising and accessible in a couple of years. Let us illustrate the interplay of flavor symmetries and SUSY in a relatively extreme case 14 For an underlying Abelian horizontal symmetry for observed quark mass and mixing hierarchy pattern, 1-3 and 2-3 mixings in $d_{R}$ sector are naturally the largest, and likewise for $\tilde{d}_{R}$ with SUSY. Cabibbo (1-2) mixing must come from up sector because of $\varepsilon$ and $\varepsilon^{\prime}$ constraints. One would have to accept $\mathrm{TeV}$ scale squarks and gluinos, but

$\star B_{d^{-}} \bar{B}_{d}, B_{s}-\bar{B}_{s}$ and $D^{0}-\bar{D}^{0}$ mixing all have common source

$\Longrightarrow$ strength and CP patterns different from SM! 
$\star B \rightarrow \rho \gamma, \omega \gamma$ : More detectable (vs. $\left.K^{* 0} \gamma\right)$ mixing-dep. CP asymmetries. Thus, the B system probes New Physics even when elusive at colliders.

\section{The Next 3 Years: A Sampler}

B Factories will reach $10^{34}$, and Tevatron Run-II will have $20 \times$ Run-I data during 2001-2003. Clearly the next 3 years would be even more exciting than the last 3: $>10^{8} B \bar{B}$ by 2003 , and comparable jump at Tevatron!

What could be revealed?

$\gamma\left(\right.$ or $\left.\phi_{3}\right)$ Program : Model-indep. methods (e.g. $\left.R_{*}\right)$ vs. "Global Fit".

Question of model indep. vs. stat. power; both can be PQCD-improved.

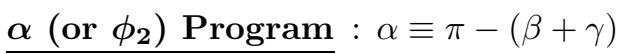

Since $\bar{B}^{0} \rightarrow \pi^{+} \pi^{-}$(direct: $\gamma$ in $V_{u b}^{*}$ ) and $\bar{B}^{0} \rightarrow B^{0} \rightarrow \pi^{+} \pi^{-}$(mixing: $\beta$ ) interfere, mixing-dep. CP probes $\alpha$. However, "P-pollution" severe.

$\Longrightarrow$ Two Paths: $\pi^{+} \pi^{-}$and $\pi^{0} \pi^{0}$ plus isospin analysis $\pi^{+} \pi^{-} \pi^{0}\left(\rho^{ \pm} \pi^{\mp}, \rho^{0} \pi^{0}\right)$ Dalitz plot analysis

This is an area where a lot of new development is expected.

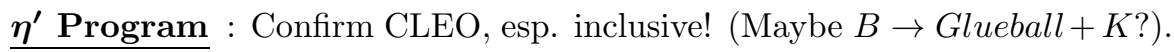

Direct CP Asymmetries : $a_{\mathrm{CP}}$ sensitivity down to few $\%$.

$\Longrightarrow$ New impact and info on/for theory. (Perhaps just testing FSI...?)

$\boldsymbol{\beta}$-crosscheck/NP probe : Mixing-dep. CP study in

$\star B \rightarrow \phi K_{S}:$ I Pure P $(s \bar{s} s)$ w/o SM phase $\Rightarrow \beta_{\phi K_{S}} \neq \beta_{\psi K_{S}}$ means NP.

$\star B \rightarrow \eta^{\prime} K_{S}$ : Not pure-P (has T) but possibility of NP source.

EWP \& $b \rightarrow d \gamma:$

$\star B \rightarrow K^{(*)} \ell^{+} \ell^{-}$will appear: $A_{\mathrm{FB}}$ via $\gamma-Z$ interference at $m_{b}$ scale!

$\star B \rightarrow \rho \gamma, \omega \gamma\left(\pi^{+} \pi^{-}\left(\pi^{0}\right)\right.$ : vertex $)$ vs. $K^{* 0} \gamma\left(K^{* 0} \rightarrow K_{S} \pi^{0}\right.$ no vertex $)$ $\Longrightarrow$ Good mixing-dep. CP probe: Nonzero $a_{\mathrm{CP}}^{\operatorname{mix}} \equiv$ New Physics.

D mixing : Confirm CLEO/FOCUS? $\Delta m_{D} \neq 0$ implies New Physics.

Charmless Rare Baryons $15 \rightarrow \eta^{\prime} \bar{\Lambda} p ; \gamma \bar{\Lambda} p$ ?

CLEO just reported ${ }^{16} B \rightarrow D^{*-} p \bar{n}, D^{*-} p \bar{p} \pi \sim 10^{-3} \lesssim D^{*} \pi, D^{*} \rho$ !

$\Longrightarrow \eta^{\prime} \bar{\Lambda} p ; \gamma \bar{\Lambda} p \sim 10^{-5} \gg \bar{\Lambda} p$ plausible, could be first charmless baryon! $\Lambda \rightarrow p \pi$ self-analyze spin: probe $B \rightarrow \eta^{\prime}, \gamma$ dynamics (and $C P / T$ ).

${ }^{c}$ Belle reported at Osaka a large $\phi K^{+}$signal $>10^{-5}$, in some conflict with a smaller number reported by CLEO. Note that the new CLEO number is above their previous upper limit. 


\section{Conclusion}

We have witnessed the riches of rare B decays from the past on weak dynamics, weak phases, new physics, and strong interaction. The highlights have been: $B \rightarrow K^{*} \gamma, K+n \pi+\gamma ; \eta^{\prime} K, \eta^{\prime}+K+n \pi ; K \pi / \pi \pi$.

The timeline is illustrated as follows:

\begin{tabular}{ccll} 
Time & $\# B \bar{B}$ & Discovery & Significance \\
\hline 1986 & $10^{5}$ & $B-B$ Mixing & $! !$ \\
1993 & few $10^{6}$ & $B \rightarrow K^{*} \gamma, X_{s} \gamma$ & EMP \\
$1997-2000$ & $10^{7}$ & $B \rightarrow \eta^{\prime} K, \eta^{\prime} X_{s}, K \pi, \phi K \ldots$ & Strong $b \rightarrow s$ P \\
& & $B \rightarrow \rho \pi, \omega \pi, \pi \pi$ & $b \rightarrow u$ T \& P \\
$2000-2003$ & $10^{8}$ & $\phi_{1} / \beta, \phi_{3} / \gamma, \phi_{2} / \alpha(?)$ & Unitarity $\triangle$ \\
& & Direct $a_{\mathrm{CP}}$ & FSI, or $\ldots ?$ \\
& & $B \rightarrow K^{(*)} \ell^{+} \ell^{-}$ & EWP \\
& & $B \rightarrow \rho \gamma, \omega \gamma$ & $b \rightarrow d$ EMP \\
& & $\ldots \ldots$ & New Physics!?
\end{tabular}

With advent of B Factories, we expect an order or more jump in number of $B \bar{B}$ 's, boosted. Detectors now have good PID plus vertexing. Boom time lies ahead - Era of BaBar/Belle/CLEO competition, and with Tevatron.

\section{References}

1. W.S. Hou, R.S. Willey and A. Soni, Phys. Rev. Lett. 58, 1608 (1987).

2. W.S. Hou and R.S. Willey, Phys. Lett. B 202, 591 (1988).

3. W.S. Hou, A. Soni and H. Steger, Phys. Rev. Lett. 59, 1521 (1987).

4. A. Golutvin, plenary talk at ICHEP2000, Osaka, Japan.

5. D. Atwood and A. Soni, Phys. Lett. B 405, 150 (1997).

6. W.S. Hou and B. Tseng, Phys. Rev. Lett. 80, 434 (1998).

7. For a little more, see my Osaka 2000 talk: G.W.S. Hou, hep-ph/0009197.

8. N.G. Deshpande et al., Phys. Rev. Lett. 82, 2240 (1999).

9. X.G. He, W.S. Hou and K.C. Yang, Phys. Rev. Lett. 83, 1100 (1999);

W.S. Hou and K.C. Yang, Phys. Rev. D 61, 073014 (2000).

10. W.S. Hou and K.C. Yang, Phys. Rev. Lett. 84, 4806 (2000).

11. W.S. Hou, J.G. Smith and F. Würthwein, e-print hep-ex/9910014.

12. X.G. He, W.S. Hou and K.C. Yang, Phys. Rev. Lett. 81, 5738 (1998).

13. C.K. Chua, X.G. He and W.S. Hou, Phys. Rev. D 60, 014003 (1999).

14. C.K. Chua and W.S. Hou, e-print hep-ph/0005015.

15. W.S. Hou and A. Soni, e-print hep-ph/0008079.

16. S. Anderson et al. (CLEO Collaboration), e-print hep-ex/0009011. 